\title{
Transition region small-scale dynamics as seen by SUMER on SOHO
}

\author{
L. Teriaca ${ }^{1}$, D. Banerjee ${ }^{2}$, A. Falchi ${ }^{3}$, J. G. Doyle ${ }^{4}$, and M. S. Madjarska ${ }^{1,5}$ \\ 1 Max-Planck-Institut für Sonnensystemforschung ${ }^{\star}$, Max-Planck-Str. 2, 37191 Katlenburg-Lindau, Germany \\ e-mail: teriaca@linmpi.mpg.de \\ 2 Indian Institute of Astrophysics, Koramangala, Bangalore 560034, India \\ 3 INAF - Osservatorio Astrofisico di Arcetri, Largo E. Fermi 5, 50125 Firenze, Italy \\ 4 Armagh Observatory, College Hill, Armagh BT61 9DG, UK \\ 5 Department of Solar Physics, Royal Observatory of Belgium, Av. Circulaire 3, 1180 Bruxelles, Belgium
}

Received 24 March 2004 / Accepted 10 August 2004

\begin{abstract}
High spectral, spatial and temporal resolution UV observations of the quiet Sun transition region show a highly structured and dynamical environment where transient supersonic flows are commonly observed. Strongly non-Gaussian line profiles are the spectral signatures of these flows and are known in the literature as explosive events. In this paper we present a high spatial resolution $\left(\approx 1^{\prime \prime}\right)$ spectroheliogram of a $273^{\prime \prime} \times 291^{\prime \prime}$ area of the quiet Sun acquired with SUMER/SOHO in the O VI spectral line at $\lambda 103.193 \mathrm{~nm}$. The extremely high quality of these observations allows us to identify tens of explosive events from which we estimate an average size of $1800 \mathrm{~km}$ and a birthrate of $2500 \mathrm{~s}^{-1}$ over the entire Sun. Estimates of the kinetic and enthalpy fluxes associated with these events show that explosive events are not important as far as solar coronal heating is concerned. The relationship with the underlying photospheric magnetic field is also studied, revealing that explosive events generally occur in regions with weak (and, very likely, mixed polarity) magnetic flux. By studying the structure of upward and downward flows exceeding those associated to average quiet Sun profiles, we find a clear correlation between the "excess" flows and the magnetic network. However, although explosive events are always associated with flow patterns often covering areas larger than the explosive event itself, the contrary is not true. In particular, almost all flows associated with the stronger concentrations of photospheric magnetic flux do not show non-Gaussian line profiles. In some cases, non-Gaussian line profiles are associated with supersonic flows in small magnetic loops. The case of a small loop showing a supersonic siphon-like flow of $\approx 130 \mathrm{~km} \mathrm{~s}^{-1}$ is studied in detail. This is, to our knowledge, the first detection of a supersonic siphon-like flow in a quiet Sun loop. In other cases, the flow patterns associated with explosive events may suggest a relation with UV spicules.
\end{abstract}

Key words. Sun: transition region - Sun: UV radiation - line: profiles

\section{Introduction}

Extreme-UltraViolet (EUV) spectroscopy of plasmas at Transition Region (TR) temperatures reveals the existence of different kinds of transient phenomena. Highly non-Gaussian line profiles showing strong Doppler shifts are frequently observed in quiet Sun areas and within coronal holes in lines formed between $6 \times 10^{4} \mathrm{~K}$ and $7 \times 10^{5} \mathrm{~K}$. First observed by Brueckner \& Bartoe (1983) in HRTS spectra, they are known as UV explosive events (EEs). Their birthrate on the quiet Sun was estimated to be around $1 \times 10^{-16} \mathrm{~m}^{-2} \mathrm{~s}^{-1}$ by Dere et al. (1989). EEs are characterised by spatial scales of $\approx 1600 \mathrm{~km}$, average lifetime of about $60 \mathrm{~s}$ and line profiles showing Doppler shifts up to $225 \mathrm{~km} \mathrm{~s}^{-1}$ (Dere et al. 1989). EEs are generally observed along the magnetic network at the

\footnotetext{
^ Former Max-Planck-Institut für Aeronomie.
}

boundaries of the super-granulation cells but away from the larger flux concentrations (Porter \& Dere 1991), in regions with weak and mixed polarity fluxes (Chae et al. 1998) and in association with episodes of photospheric magnetic flux cancellation (Dere et al. 1991; Chae et al. 1998; Ryutova \& Tarbell 2000). Furthermore, the spectral signature of EEs shows enhancements in both the red and blue wings of the profile, implying high velocity flows similar to a bi-directional jet associated with magnetic reconnection (Innes et al. 1997). Hence, EEs are believed to be the product of magnetic reconnection, a magnetic cancellation of the photospheric fields with emerging flux (Dere et al. 1991) or the interaction between separate flux elements driven together by convective motions (Porter \& Dere 1991). The large velocities associated with EEs suggest that kinetic and enthalpy flows could be relevant to the coronal energy budget. Winebarger et al. (2002) estimated the global energy 
release associated with explosive events through an analysis of the velocity differential emission measure. They show that EEs can provide an energy flux $\approx 40 \mathrm{~W} \mathrm{~m}^{-2}$, implying that the EEs themselves are not energetically significant to the solar atmosphere.

More recently another class of transient events, named blinkers (e.g., Harrison 1997; Harrison et al. 1999; Bewsher et al. 2002), was discovered and studied with the CDS spectrometer (Harrison et al. 1995) aboard SOHO. Blinkers are defined as enhancements of the radiance of lines formed at mid-TR temperatures (e.g., O v $\lambda 62.97 \mathrm{~nm}$, formation temperature of $2.5 \times 10^{5} \mathrm{~K}$ ) primarily occurring at network boundaries (Harrison et al. 1999; Bewsher et al. 2002), although they have been also observed in the internetwork (Brković et al. 2001). Due to the lower spatial and spectral resolution of CDS with respect to SUMER, it has been a matter of debate whether blinkers are EEs seen at lower resolution or whether they are different phenomena. Although it seems very likely that some events, classified as short-duration blinkers, could just be large EEs, recent studies seem to support the idea that blinkers and EEs are different phenomena (Chae et al. 2000; Peter \& Brković 2003; Madjarska \& Doyle 2003). The latter study, in particular, shows that blinkers as seen in SUMER data have at most flows of $\approx 25 \mathrm{~km} \mathrm{~s}^{-1}$.

Although EEs can be observed up to $2^{\prime \prime}-3^{\prime \prime}$ above the visible limb (Dere 1992), both EEs and blinkers are essentially observed on the disk. Going off-limb, UV observations reveal another type of dynamic transients: UV spicules. UV spicules are elongated structures extending above the solar limb up to $\approx 15 \mathrm{Mm}\left(\approx 21^{\prime \prime}\right.$, at the SOHO-Sun distance $\left.1^{\prime \prime} \approx 715 \mathrm{~km}\right)$ (Withbroe 1983; Cook et al. 1984), showing variations indicative of apparent motions around $30 \mathrm{~km} \mathrm{~s}^{-1}$ (Wilhelm 2000). Whether UV spicules are cospatial or the extension of the long known H $\alpha$ spicules (extensively reviewed by Beckers 1968, 1972; Bray \& Loughhead 1974; Athay 1976; Suematsu 1998) is still uncertain, although there are strong indications that a close relation may exist (see Wilhelm 2000, and references therein). Moreover, it is still not known whether they have any relation with other UV dynamic events. Wilhelm (2000) suggested that EEs and spicules may be closely related, outlining a mechanism where EEs could be the first stage of a sequence of events leading to the formation of a spicule.

In this paper we present high spatial resolution observations of a large quiet Sun area in the mid-transition region line O VI $\lambda 103.193 \mathrm{~nm}\left(3 \times 10^{5} \mathrm{~K}\right)$. These observations allow us to identify tens of explosive events and to investigate their dynamical characteristics and their relationship with the magnetic network. The birthrate and the typical size of these events are also found. From our results we make an estimation of the total energy (kinetic energy plus enthalpy) flux associated with EEs. A detailed analysis of the mass flows exceeding those associated to average quiet Sun profiles is performed and a possible link between EEs and spicules is explored. The first, to our knowledge, detection of a supersonic flow in a small loop in the quiet Sun is also studied in detail. The relevance of our results to the understanding of the structuring of the solar TR is discussed.

\section{Observations}

A raster of a $273^{\prime \prime} \times 291^{\prime \prime}$ quiet region around Sun centre (see Fig. 1) was obtained on 30 January 1996 by the SUMER spectrograph (Wilhelm et al. 1995) aboard SOHO in the O VI $\lambda 103.193 \mathrm{~nm}$ line. The capabilities of the SUMER experiment are extensively described by Wilhelm et al. (1997) and Lemaire et al. (1997). The raster starts at 04:38:01 and ends at 04:57:03 UTC. It consists of a series of spectra obtained exposing for $3 \mathrm{~s}$ the central part of detector A. After each exposure only 50 of the 1024 spectral pixels of the detector were transmitted to the ground. This, with a spectral resolution of $4.418 \times 10^{-3} \mathrm{~nm}$ per pixel, corresponds to a spectral window of $\approx 0.22 \mathrm{~nm}$. The observed area was covered by stepping the $1^{\prime \prime} \times 300^{\prime \prime}$ slit westward $0.76^{\prime \prime}$ a total of 360 times. The reduction of SUMER data requires several stages. After correcting for dead time and local gain depression, data were flat-fielded and destretched using the provided software. Radiometrically calibrated spectral and line radiances have been obtained by using the RADIOMETRY.PRO routine. To give an indication of the noise level, spectral and line radiances are also shown in total count per pixel.

Around 25 min later, 15 full disk magnetograms were acquired with MDI (Scherrer et al. 1995) from 05:22 to 05:38 UTC. No relevant variation is observed during the $16 \mathrm{~min}$ separing the first and the last magnetogram. After correcting for the solar rotation, an average of the 15 frames was obtained, allowing us to build a good signal-to-noise magnetogram to superpose to the O VI observations. The resulting magnetogram was aligned to the SUMER raster by cross-correlating the absolute magnetic flux and the SUMER continuum around $103.08 \mathrm{~nm}$, which forms in the middle chromosphere (around 1.1 Mm above $\tau_{5000}=1$, according to model $\mathrm{C}$ of Vernazza et al. 1981). At each spatial location, the continuum level was obtained by averaging the spectral radiances over the $10 \mathrm{spec}-$ tral pixels between 103.062 and $103.106 \mathrm{~nm}$. The latter point is away from the $\mathrm{O}$ VI line centre by $\approx 4$ times the average $F W H M$ of the line. The alignement is estimated to be precise within $3^{\prime \prime}$.

A logarithmically-scaled image of the observed area obtained by integrating over the OVI $\lambda 103.193 \mathrm{~nm}$ line is shown in Fig. 1. Levels of the longitudinal magnetic flux of (10, 25 and 40) $\mathrm{G}$ are shown with white (positive polarity) and black (negative polarity) solid lines. The structures visible in Fig. 1 and their relationship with the underlying longitudinal magnetic flux have been discussed in detail by Warren \& Winebarger (2000). Here, we want to focus on a detailed analysis of the line profiles in order to study the small-scale dynamics of the transition region plasma. In this way we further exploit the potentials of one of the best raster sequences taken by the SUMER spectrograph aboard SOHO.

\section{Explosive events identification and general characteristics}

The profile of a line emitted by an optically-thin plasma is only determined by the dynamics of the emitting ions (neglecting the small atomic broadening). Motions on scales larger than the instrumental spatial resolution will lead to the shift of the entire line profile, while thermal and non-thermal motions on scales 


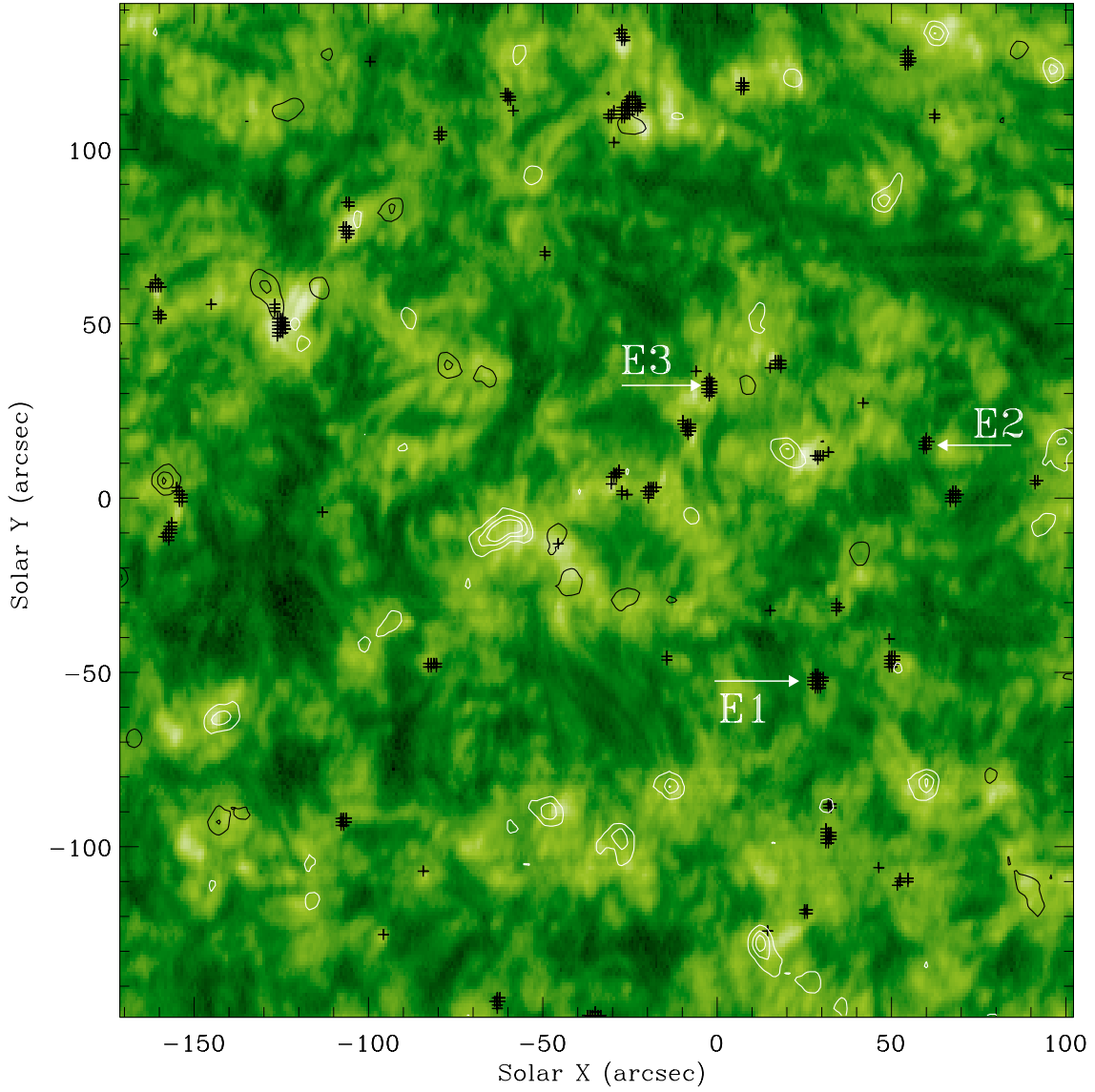

Fig. 1. Logarithmically-scaled image of the quiet Sun obtained by integrating over the $\mathrm{O}$ VI $\lambda 103.193 \mathrm{~nm}$ line $\left(3 \times 10^{5} \mathrm{~K}\right)$ after subtracting the continuum. The minimum and the maximum of the image correspond to radiances around 14 and $4654 \mathrm{~mW} \mathrm{~m}^{-2} \mathrm{sr}^{-1}$, respectively. Levels of the longitudinal magnetic flux of $10 \mathrm{G}, 25 \mathrm{G}$ and $40 \mathrm{G}$ are shown with white (positive) and black (negative polarity) solid lines. The locations where non-Gaussian line profiles were found are marked with a black + . smaller than the spatial resolution will determine the shape of the profile. Moreover, different structures with different dynamics may be present along the line of sight (LOS) over which the line is integrated. However, quiet Sun profiles are generally well represented by a single Gaussian (although a combination of two Gaussians may represent better the "quiet" TR line profile; see, e.g., Peter 2001), indicating that the small-scale thermal and non-thermal motions both follow a Maxwellian distribution in most of the cases. In this framework, the triggering of high-speed flows will lead to the non-Gaussian line profiles characterising the explosive events. It should be noted that flows on scales larger than the spatial resolution may also lead to non-Gaussian line profiles when their emission is combined with that from other structures along the LOS.

To identify explosive event profiles, a single Gaussian has been fitted to all the $\approx 10^{5}$ spectra forming the raster. All line profiles, for which at least one of the fitted parameters (or the $\chi^{2}$ ) was diverging by more than $3 \sigma$ from the average of its distribution, were first selected. All the profiles forming this sub-sample were, hence, visually inspected and all profiles for which at least three to four contiguous pixels were consistently diverging by a Gaussian profile (by more than their Poissonian uncertainties) were flagged as explosive events. The positions of the flagged profiles are indicated by black + marks on the radiance image displayed in Fig. 1.

It is clearly visible that the selected points are not randomly distributed, but appear to form small patches. Considering all contiguous points as belonging to the same event, it is possible to count around $N_{\mathrm{EE}}=50$ explosive events in the observed area with an average size of $\approx 1800 \mathrm{~km}$. As representative examples we show in Fig. 2 single-pixel line profiles from three locations marked as E1, E2, and E3 in Fig. 1. The majority $(\approx 2 / 3)$ of the profiles show a stronger blue wing with average bulk velocities $|v| \geq 100 \mathrm{~km} \mathrm{~s}^{-1}$. The average radiance $L$ at the EEs locations is $1.08 \mathrm{~W} \mathrm{~m}^{-2} \mathrm{sr}^{-1}$, compared to a value of $0.37 \mathrm{~W} \mathrm{~m}^{-2} \mathrm{sr}^{-1}$ obtained by averaging over the whole dataset.

Once all the explosive events were identified, it is possible to estimate their rate of occurrence. The explosive events birthrate, $R$, is given by

$$
R=\frac{N_{\mathrm{EE}}}{t_{\mathrm{exp}} A_{\mathrm{obs}}},
$$

where $t_{\mathrm{exp}}$ is the exposure time, $A_{\mathrm{obs}}$ the rastered area and $N_{\mathrm{EE}}$ is the number of observed explosive events. Given an area of $4.1 \times 10^{16} \mathrm{~m}^{2}\left(273^{\prime \prime} \times 291^{\prime \prime}\right), t_{\mathrm{exp}}=3 \mathrm{~s}$ and $N_{\mathrm{EE}}=50$, we obtain an event birthrate $R=4.1 \times 10^{-16} \mathrm{~m}^{-2} \mathrm{~s}^{-1}\left(\simeq 2500 \mathrm{~s}^{-1}\right.$ over the whole Sun).

Although explosive events leave a weak signature in chromospheric lines (Teriaca et al. 2002; Madjarska \& Doyle 2002), no change is observable in lines formed at $T \geq 1 \mathrm{MK}$ (Teriaca et al. 2002; Doyle et al. 2004). This suggests that, at sites of strong velocities, the plasma is not heated to coronal temperatures, indicating that energy is mostly used to accelerate it. The presence of upward velocities $|v| \geq 100 \mathrm{~km} \mathrm{~s}^{-1}$ would, within this framework, suggest a possible role for kinetic and enthalpy energy fluxes associated with explosive events in heating the quiet Sun corona. 

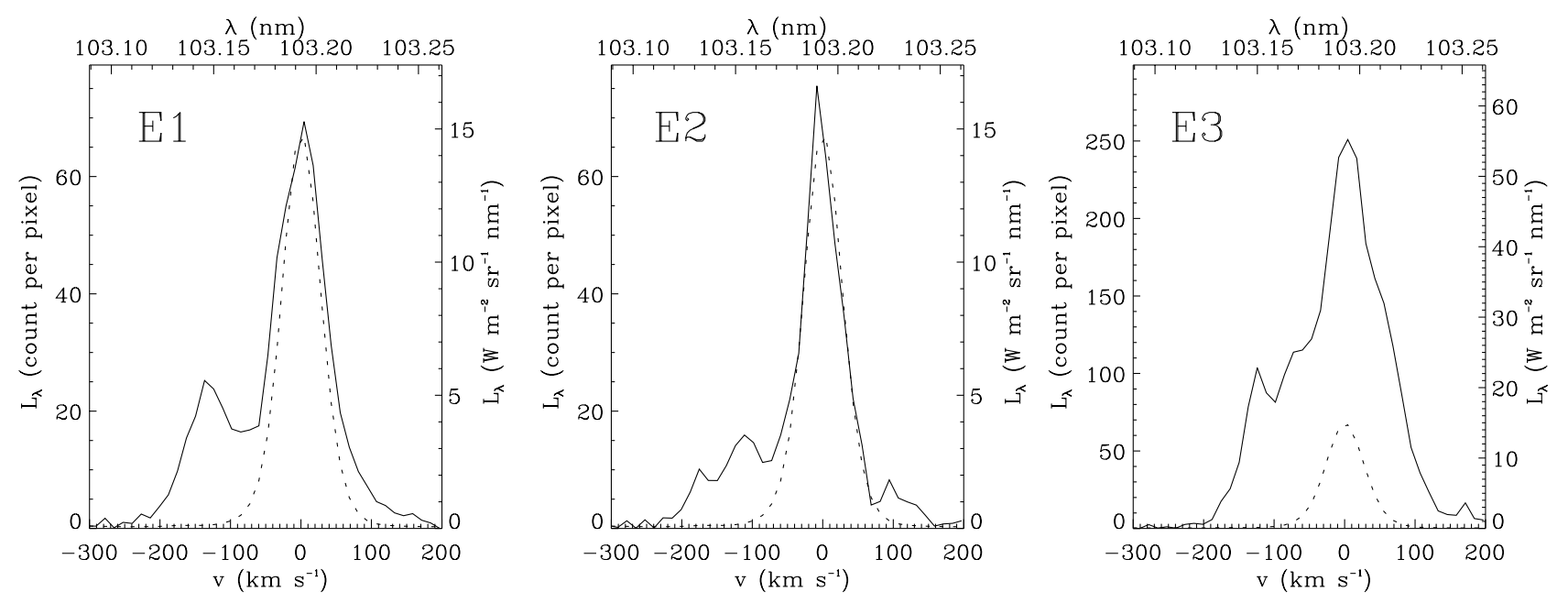

Fig. 2. Spectral radiances, $L_{\lambda}$, at the centre of three of the explosive events identified in Fig. 1 . The profile obtained by averaging the entire dataset is shown by the dotted lines.

An (order of magnitude) estimate of the kinetic energy associated with an explosive event, can be written as:

$E_{\text {kin }}=0.5 m_{\mathrm{H}} N_{\mathrm{e}} f v^{2}|v| A \tau$

where $m_{\mathrm{H}}$ is the mass of the hydrogen atom, $N_{\mathrm{e}}$ the electron density $\left(N_{\mathrm{e}}=N_{\mathrm{H}}\right.$, fully ionised plasma of pure $\left.\mathrm{H}\right)$ and $v$, $A$ and $\tau$ are the typical speed, area and lifetime of explosive events, respectively. The volumetric filling factor, $f$, accounts for the filamentary structure of the transition region and is estimated to be around 0.01 (Feldman et al. 1979). However, for a large explosive event, Dere (1992) found a value of $10^{-4}$ by comparing the electron density derived from the ratio of density sensitive lines within the $\lambda 140 \mathrm{~nm}$ O IV multiplet with that obtained from the volumetric emission measure. Applying the same technique to another explosive event observed in the same O IV multiplet by Teriaca et al. (2001), we find a value $\approx 0.007$ for $f$. These values also show that EE-like line profiles are due to motions on scales much smaller than the actual spatial resolution. Let us consider $A=3.2 \times 10^{12} \mathrm{~m}^{2}\left(1800^{2} \mathrm{~km}^{2}\right)$ and $|v|=100 \mathrm{~km} \mathrm{~s}^{-1}$ from the present analysis, $\tau=60 \mathrm{~s}$ (Dere et al. 1989) and the value 0.007 for the filling factor $f$. Teriaca et al. (2001) find values around $2 \times 10^{10} \mathrm{~cm}^{-3}$ for the electron density during EEs observed in $\mathrm{O}$ IV lines (formed around $1.7 \times 10^{5} \mathrm{~K}$ ). Assuming constant pressure through the TR, we obtain an electron density of $1 \times 10^{10} \mathrm{~cm}^{-3}$ for the plasma around $3 \times 10^{5} \mathrm{~K}$. Adopting this value of $N_{\mathrm{e}}$, Eq. (2) yields a kinetic energy $E_{\text {kin }}=1 \times 10^{16} \mathrm{~J}$ for a typical event.

Similarly, the enthalpy energy flux is:

$H=5 N_{\mathrm{e}} K_{\mathrm{B}} T f|v| A \tau$

where $K_{\mathrm{B}}$ is the Boltzmann constant and $T$ the line formation temperature. Considering $T=3 \times 10^{5} \mathrm{~K}$ (O VI), we obtain $H=3 \times 10^{16} \mathrm{~J}$. Finally, the total energy flux associated with explosive events is obtained by multiplying the typical energy for one event and the birthrate: $E_{\mathrm{Tot}}=\left(E_{\mathrm{kin}}+H\right) \times R=20 \mathrm{~W} \mathrm{~m}^{-2}$. This value (provided $f$ is correctly estimated) is at least one order of magnitude smaller than the quiet Sun radiative losses for all plasmas hotter than $3 \times 10^{5} \mathrm{~K}\left(\approx 400 \mathrm{~W} \mathrm{~m}^{-2}\right)$ calculated by
Dere \& Mason (1993). Furthermore, the energy flux should be further reduced by considering that not all the explosive events show a dominant blueshifted (upward directed) component.

\section{Relation with the magnetic network and structure of the velocity field}

From the analysis of Fig. 1 it appears that the observed events outline the network (as seen in $\mathrm{O} \mathrm{VI}$ ) but generally do not appear in the brightest regions. Moreover, they seem to avoid the areas where the longitudinal magnetic flux is stronger. In fact, for all but two of these events, the average absolute longitudinal flux in the underlying area is below $6 \mathrm{G}$. Although a vector magnetogram with higher spatial resolution would be necessary to measure the true magnetic flux, these observations indicate explosive events to occur in regions away from the strong magnetic field concentrations.

However, at the pressure characteristic of the network midtransition region $\left(n_{\mathrm{e}} T_{\mathrm{e}}=7 \times 10^{14} \mathrm{~cm}^{-3} \mathrm{~K}\right.$, Teriaca et al. 2001), the low- $\beta$ assumption holds also for magnetic fields of a few gauss and the plasma is forced to move along the magnetic field lines. In this framework, the study of plasma flows can provide information about the structuring of the transition region and the configuration of the magnetic field also well above the photosphere, where direct measurements are, thus far, not feasible.

In the previous section only the clearly non-Gaussian line profiles were marked as belonging to explosive events. Profiles characterised by large broadenings and/or line shifts but still retaining a Gaussian shape were not considered. However, these profiles could still indicate relevant mass flows and/or smallscale motions that exceed those generally observed on the quiet Sun. To identify these "excess motions" the following quantity was calculated for each spectrum of coordinates $(i, j)$ forming the raster.

$D_{i, j}(v)= \begin{cases}f_{i, j}(v)-\bar{f}_{i, j}(v) & \left.\text { (if } f_{i, j}(v)-\bar{f}_{i, j}(v)>0\right) \\ 0 & \text { (otherwise) }\end{cases}$ 


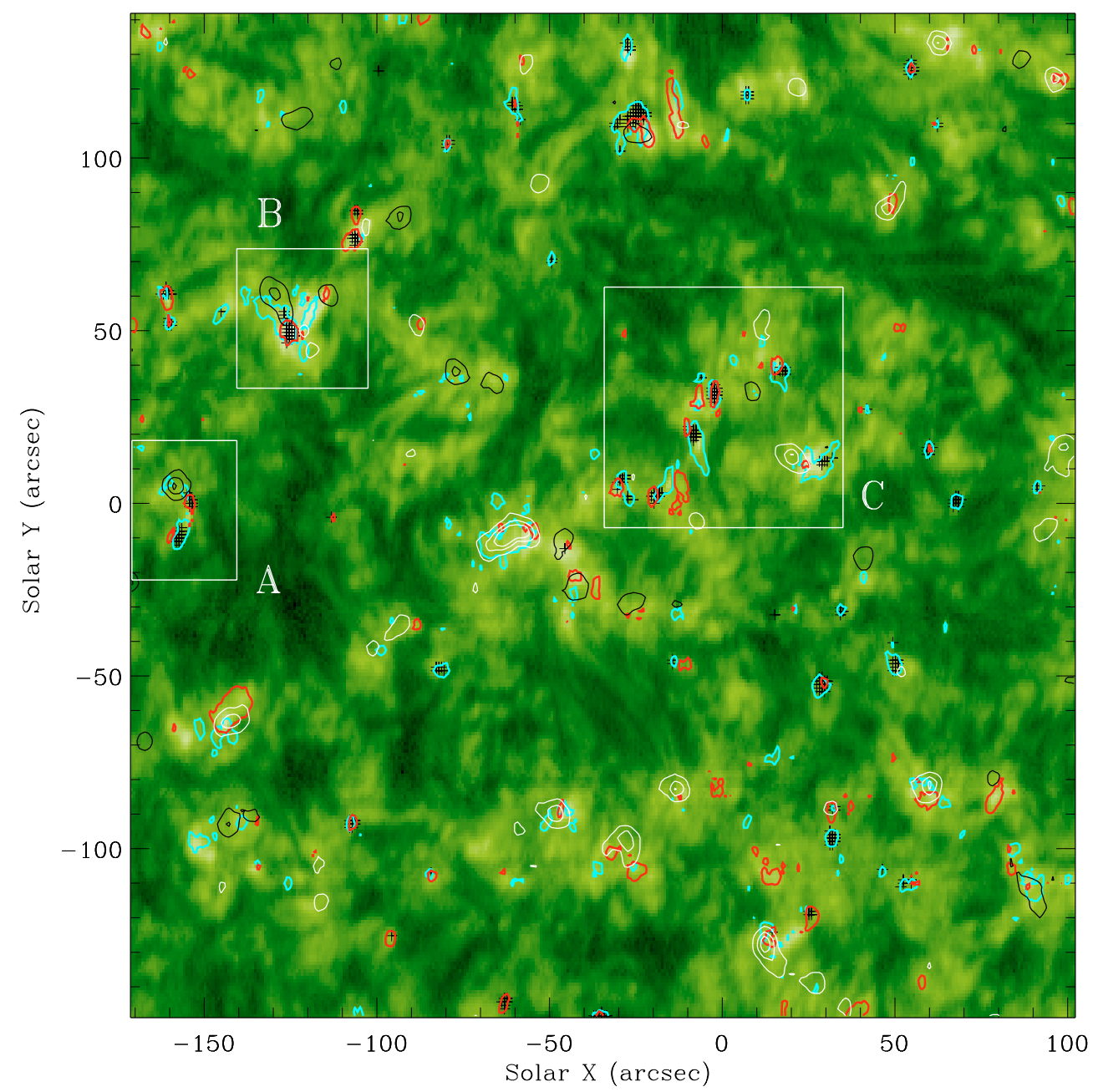

Fig. 3. Radiance image as in Fig. 1. Solid dark-grey and light-grey (red and light-blue in the electronic version) isocontours show the downward and upward mass fluxes, respectively. The contours are traced for levels of $\left\langle\overline{F^{\mathrm{B}, \mathrm{R}}}+3 \sigma\left(F^{\mathrm{B}, \mathrm{R}}\right)\right\rangle=576$ ( count $\left.^{1 / 2} \mathrm{~km} \mathrm{~s}^{-1}\right)$. Levels of the longitudinal magnetic flux of (10, 25 and 40) $\mathrm{G}$ are shown with white (positive polarity) and black (negative polarity) solid lines. The locations where nonGaussian line profiles were found are marked with a black + .

where $\bar{f}_{i, j}(v)$ is the spectrum obtained averaging over the whole dataset and scaled to match the observed peak amplitude at position $(i, j)$.

The mass flux of a plasma of density $\rho$ moving through an area $A$, with a velocity $v$ normal to $A$, is given by $\rho v A \propto$ $N_{\mathrm{e}} v A$. Assuming the pixel size as the unit area and considering that for an allowed transition in an optically thin plasma $N_{\mathrm{e}}$ is proportional to the square-root of the line radiance $L$, the mass flux can be written as $\propto \sqrt{L} \cdot v$. For each spectrum of the raster (of coordinates $i, j$ ), the upward and downward directed mass fluxes $\left(F^{\mathrm{B}}\right.$ and $F^{\mathrm{R}}$, respectively) were computed as:

$F_{i, j}^{\mathrm{B}, \mathrm{R}}=\frac{\int_{v_{a}}^{v_{b}} v D_{i, j}(v) \mathrm{d} v}{\left[\int_{v_{a}}^{v_{b}} D_{i, j}(v) \mathrm{d} v\right]^{1 / 2}}$

where

$\begin{cases}F_{i, j}^{\mathrm{B}}: v_{a}=-200, & v_{b}=0 \\ F_{i, j}^{\mathrm{R}}: v_{a}=0, & v_{b}=200 .\end{cases}$

The value of the speed in the blue and red wings, $v^{\mathrm{B}}$ and $v^{\mathrm{R}}$, can be easily calculated as the first moment of the difference spectrum $D_{i, j}(v)$. For both distributions the average $\overline{F^{\mathrm{B}, \mathrm{R}}}$ and the standard deviation $\sigma\left(F^{\mathrm{B}, \mathrm{R}}\right)$ were also calculated. The quantities $\overline{F^{\mathrm{B}}}+3 \sigma\left(F^{\mathrm{B}}\right)$ and $\overline{F^{\mathrm{R}}}+3 \sigma\left(F^{\mathrm{R}}\right)$ are quite similar being equal to 611 and 541 (count ${ }^{1 / 2} \mathrm{~km} \mathrm{~s}^{-1}$ ), respectively. Finally, the speed and the mass flux are considered as reliable only at those locations were the radiance of the difference spectrum is 2.3 times larger than the uncertainties in the radiance of the wing itself. In Fig. 3, the isocontours at the $\left\langle\overline{F^{\mathrm{B}, \mathrm{R}}}+3 \sigma\left(F^{\mathrm{B}, \mathrm{R}}\right)\right\rangle=$ 576 level are shown with dark-grey $\left(F^{\mathrm{R}}\right)$ and light-grey $\left(F^{\mathrm{B}}\right)$ thick solid lines (red and light-blue in the electronic version). From the analysis of Fig. 3 several facts appear evident. First, a good correlation between the "excess" mass flows and the magnetic field is clearly present. However, the flow patterns associated with the stronger concentrations of the magnetic flux generally does not show any non-Gaussian (or EE-like) line profile. Second, EEs are associated with flow patterns often covering areas larger than those showing strongly nonGaussian line profiles and occur in areas with weak (and, very likely, mixed polarity) magnetic flux. 


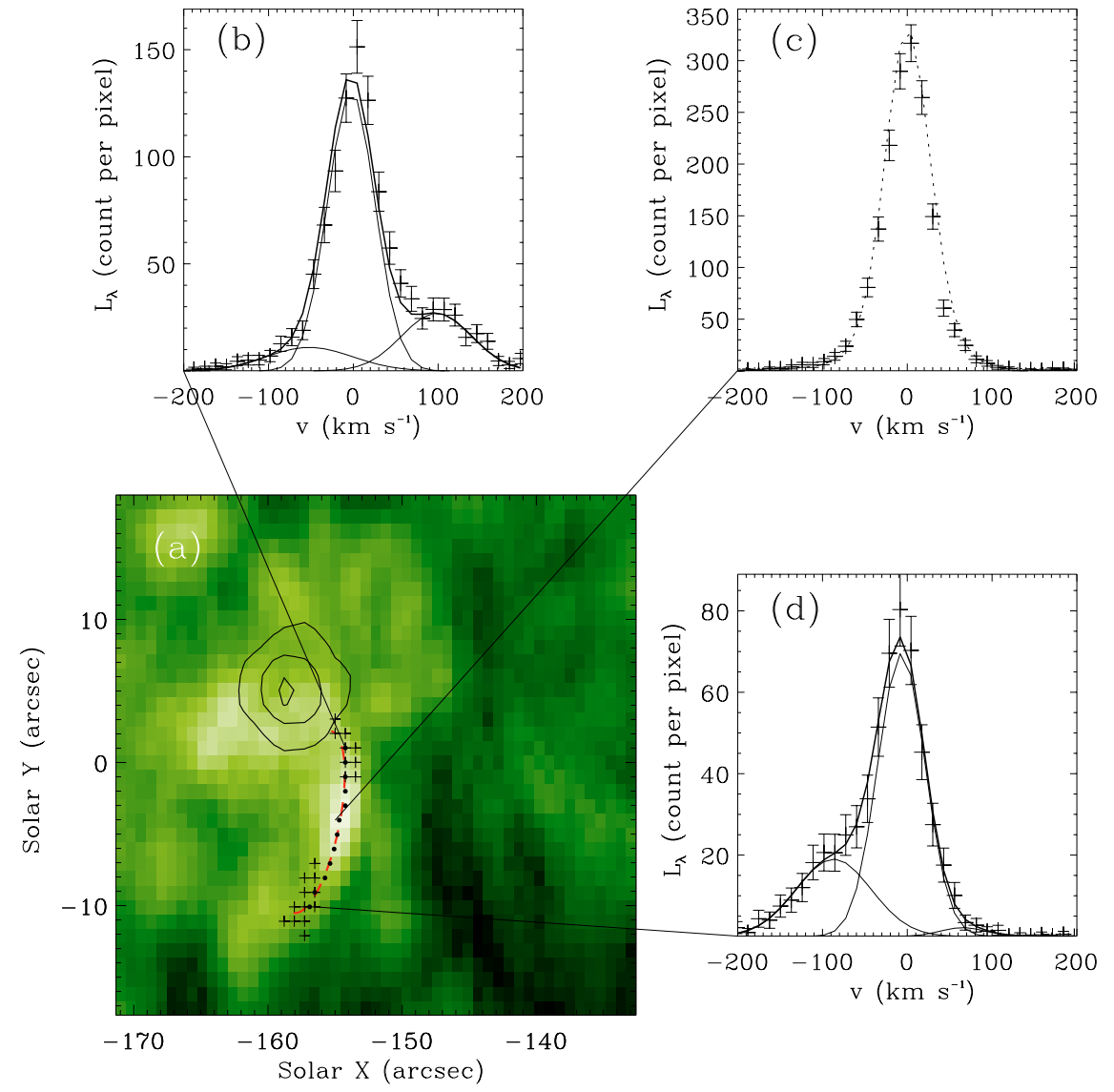

Fig. 4. Detailed view of box A in Fig. 1 together with the line profiles at the indicated locations. a) Logarithmically-scaled radiance image. Isocontours of the negative polarity of the longitudinal magnetic flux at $(-10,-25$ and $-40) \mathrm{G}$ are shown with black solid lines (no positive flux above $10 \mathrm{G}$ is present in the displayed area). Locations where non-Gaussian line profiles were found are marked with a black + . The dashed dark-grey (red) line indicates the projection (on the plane perpendicular to the LOS) of a semi-circular loop with a diameter of $13^{\prime \prime}$. The loop is inclined by $18^{\circ}$ with respect to the LOS and the footpoint line rotated by $12.5^{\circ}$ clockwise. The black dots indicate the measured position of the observed loop. b) Line profile at a location on the northern leg of the loop. The bars indicate the data points. The thin solid lines show the three components used to fit the data while the resulting fitting profile is represented by the thick solid line. c) Line profile at the top of the loop. The dotted line shows the profile obtained averaging over the whole raster times 4.9. d) The same as for panel b) but for the southern leg of the loop.
This seems to indicate a different structure of the transition region at the locations of stronger flux concentration than in the remaining network. The large flux concentrations could be the roots of coronal funnels either open or forming the base of large coronal loops, while the remaining network is dominated by small TR loops (Dowdy et al. 1986; Peter 2001). At the regions of strong magnetic flux and large flows, a single Gaussian provides a good fit of the line profiles showing bulk velocities either downwards $\left(\approx 30 \mathrm{~km} \mathrm{~s}^{-1}\right.$ around $\left.X=-140, Y=-60\right)$ or upwards $\left(\approx-17 \mathrm{~km} \mathrm{~s}^{-1}\right.$ around $\left.X=-60, Y=-10\right)$. Heating/pressure imbalances can easily explain subsonic flows in large coronal loops (see later in the text). The EEs would, instead, be typical of small loops that do not reach coronal temperatures, in agreement with Teriaca et al. (2002) and Doyle et al. (2004) who report that EEs are not seen in lines formed at coronal temperatures. In this context it is interesting to note that the energy flux associated with EEs $\left(20 \mathrm{~W} \mathrm{~m}^{-2}\right)$, although small with respect to the energy requirements of the whole corona, it is comparable to the average energy radiated in the O VI $103.193 \mathrm{~nm}$ line during EEs: $4 \pi L=13.6 \mathrm{~W} \mathrm{~m}^{-2}$.

Now we focus our attention on the flows associated with EEs. It is important to underline here that the EEs profiles and the "excess" mass flows were studied in a different manner. The EEs were identified by their strongly non-Gaussian line profiles while the "excess" flows were determined by the moments of the difference spectra as in Eq. (5). This means that the "excess" flows with no corresponding EE-like profiles are due to a shift and/or broadening of the entire line profile, while the sign of the mass flow at the location of EEs gives information on the spatial characteristics and extension of the enhanced wings characterising such events. The majority of the EEs are dominated by upward flows, confirming that the majority of these events, when observed in O VI, show a prevalent blue wing emission, although it should be also noted the existence of few EEs showing only the red wing. In some of the cases the topology of the "excess" flows could indicate siphonlike flows in closed magnetic structures, while in other cases blue and red patches seem to originate from the same EE but extend beyond it, and the more extended blue pattern may be indicative of an association with spicules.

A particularly interesting example of flows in a closed magnetic structure is outlined by box A in Fig. 3. Figure 4a shows an enlarged view of this region, revealing a small isolated loop. Three line profiles corresponding to the two legs (panels $b$ and d) and the top (panel c) of the loop are also shown. Note that the line profile in Fig. 4b indicates the presence of downflows with velocities $\approx 100 \mathrm{~km} \mathrm{~s}^{-1}$ in correspondence with one of the legs of the loop. Whereas the line profile in Fig. 4d, corresponding to the other leg, shows the presence of upflows with velocities $\approx-100 \mathrm{~km} \mathrm{~s}^{-1}$. Furthermore, the line profile corresponding to the top portion of the loop (Fig. 4c) shows a profile very close in shape to the average quiet Sun profile, indicating no shifts at all. This is clearly a LOS effect and represents a case of supersonic flows within a magnetic loop $\left(c_{\mathrm{s}} \approx 50 \mathrm{~km} \mathrm{~s}^{-1}\right)$. To test this hypothesis further, the loop position was found by determining the positions of the maxima along horizontal cuts in the radiance image (black dots in Fig. 4a). A semi-circular geometrical loop of $13^{\prime \prime}$ diameter was then considered and it 


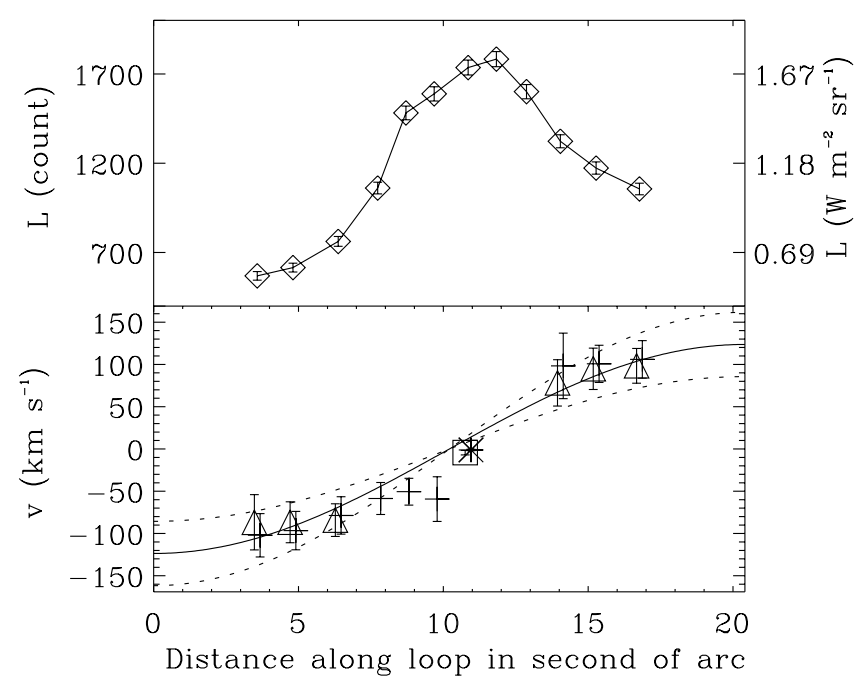

Fig. 5. In the top panel the line radiance, $L$, along the loop (following the dotted line in Fig. 4a) is shown. The distance along the loop is reckoned from the southern extreme of the loop. In the bottom panel we show the speeds obtained by the moment analysis (+ symbols) together with the measurements obtained by the multi-Gaussian fitting (triangles). Negative values indicate blueshifts (upflows). For the sake of clarity, the two sets of measurements are slightly shifted with respect to each other in abscissa. A single Gaussian fit (square) and the first moment (asterisk) of the line profile at the loop top are also shown. The solid line indicate the LOS component for a $130 \mathrm{~km} \mathrm{~s}^{-1}$ flow along the semi-circular geometrical loop whose projection on the plain perpendicular to the LOS is shown by the dark-grey (red) dashed line in Fig. 4a. The dotted lines are obtained for velocities of 90 and $170 \mathrm{~km} \mathrm{~s}^{-1}$.

was inclined with respect to the LOS, and its footpoint line rotated until its projection on the plane perpendicular to the LOS (dashed dark-grey/red line in Fig. 4a) matched the position of the observed loop. The match was obtained for an inclination of $18^{\circ}$ with respect to the LOS and a rotation of $12.5^{\circ}$ clockwise of the footpoint line.

The top panel of Fig. 5 shows the line radiance along the loop. It is interesting to note that the top of the loop is brighter than both the two legs. The lower panel of Fig. 5 shows the value of the wing speed along the loop (plus sign). Negative values indicate blueshifts (upflows). $v^{\mathrm{B}}$ (upflows) is shown when $F^{\mathrm{B}}>F^{\mathrm{R}}$ while $v^{\mathrm{R}}$ (downflows) is shown when $F^{\mathrm{R}}>F^{\mathrm{B}}$. $F^{\mathrm{B}}$ is much larger than $F^{\mathrm{R}}$ for the southern leg of the loop while the contrary holds for the northern leg. To verify the values obtained from the moment analysis of the difference spectra, a triple Gaussian fit was also performed at the locations on the legs of the loop and the results for the dominant wing are shown in the bottom panel of Fig. 5 by triangles. An example of the applied fitting is shown in Fig. $4 \mathrm{~b}$ and $4 \mathrm{~d}$. In the lower panel of Fig. 5, the speed measurements at the top of the loop were obtained from a single Gaussian fit (square) and from the first moment (asterisk) of the line profile at that location. Finally, the speed component along the LOS was calculated at each point along the geometrical loop for a flow of $130 \mathrm{~km} \mathrm{~s}^{-1}$ and the results were compared with the measured values. The agreement is remarkably good and represents (to our knowledge) the first detection of a supersonic flow in a quiet Sun loop. Other indications of similar flows can be seen in Fig. 3 around coordinates $(30,-95)$ and $(-160,55)$. It is interesting to note that all these three cases concern loop structures oriented in the north-south direction, i.e. such that the time to scan through the structure was minimum. This may be an indication that these supersonic flows are of short duration. However, observations extended in time are necessary to confirm it.

High-speed flows (50 to $100 \mathrm{~km} \mathrm{~s}^{-1}$ ) in cold loops ( $T \leq$ $5 \times 10^{5} \mathrm{~K}$ ) have been so far observed only in active regions with both CDS (Brekke et al. 1997; Kjeldseth-Moe \& Brekke 1998) and SUMER (Wilhelm 1997). Our observations show such phenomenon to exist also in the quiet Sun. The short time in which the loop in Fig. 4 was rastered $(\approx 15 \mathrm{~s})$ makes our observations much similar to a snapshot and allows direct comparison with numerical models of flows along loops. Flows can be driven by asymmetries (such as heating or pressure imbalances) between the two legs of the loop (Boris \& Mariska 1982; Mariska \& Boris 1983; McClymont \& Craig 1987; Mariska 1988; McClymont 1989; Spadaro et al. 1991; Thomas \& Montesinos 1991; Robb \& Cally 1992; Orlando et al. 1995a,b) or by radiatively-cooling condensations (Reale et al. 1996, 1997; Müller et al. 2003). In the majority of the cases the derived flows are very small, of the order of few kilometers per second. However, short duration supersonic flows may also be obtained (see, e.g. Orlando et al. 1995a,b; Robb $\&$ Cally 1992). It should be noted that a footpoint pressure imbalance would lead to a flow arising from the footpoint with the higher pressure. If we assume the ambient pressure being equal at the two footpoints, then the stronger magnetic concentration corresponds to a smaller gas pressure and the flow will be directed upwards at the footpoint where the magnetic flux is weaker and downwards at the footpoint with stronger flux concentration, as predicted by Thomas \& Montesinos (1991). This is exactly what we observe in the case illustrated in Figs. 4 and 5. This finding qualitatively resembles the siphon flow observed by Rüedi et al. (1992) in the infrared. A similar scenario may also characterise the case at $(30,-95)$.

Although the majority of the EEs visible in Fig. 3 are located in areas away from strong magnetic flux concentrations, a remarkable exception is indicated by the region within box $\mathrm{B}$ in Fig. 3. The left panel of Fig. 6 represents an enlarged view of this region. The white and black contours represent the positive and negative longitudinal magnetic flux, respectively. Thick solid dark-grey and light-grey (red and light-blue) contours show the downwards and upwards directed mass fluxes, respectively. Finally, the black + mark the location of highly non-Gaussian line profiles, an example of which is provided in the right panel. A closer inspection of Fig. 6 (left panel) reveals that the non-Gaussian profiles are located in what appears to be the intersection of two systems of loops, one connecting the positive polarity $\mathrm{P} 1$ to the negative polarity $\mathrm{N} 1$ and the other connecting P2 and N2. The flow pattern seems to be also organised along the same directions. The flow pattern and magnetic field configuration seem shifted a few second of arc with respect to the other but this may well be a residual error in superposing the magnetic field. This is a clear example of a small-scale magnetic reconnection between two loop systems. The reconnection site is indicated by the patch of non-Gaussian 

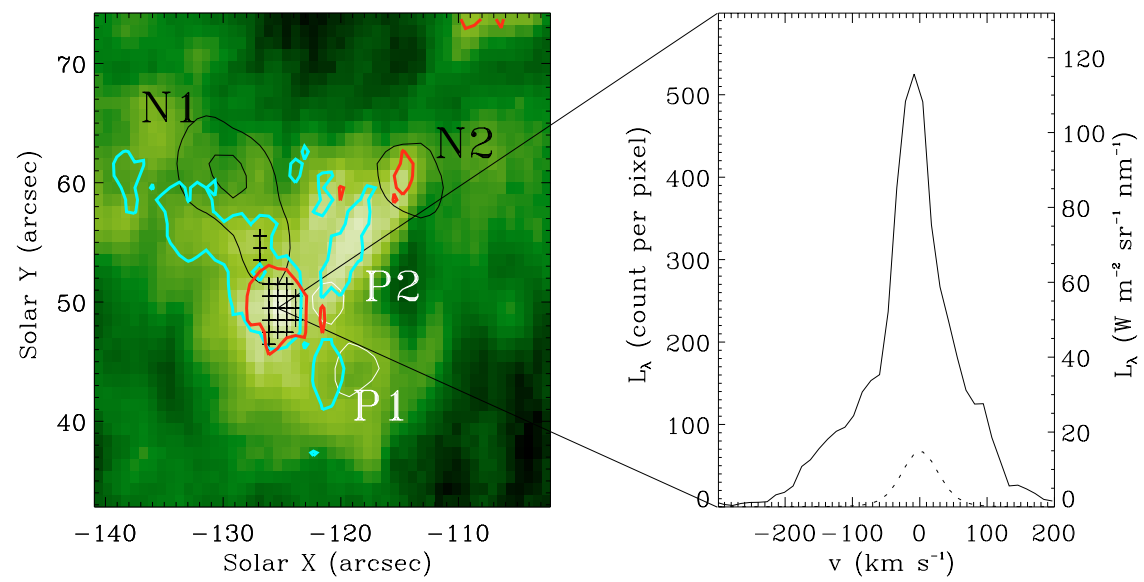

Fig. 6. Detailed view of the region marked as B in Fig. 3. On the radiance image on the left panel isocontours are defined as in Fig. 3. The locations where non-Gaussian line profiles were found are marked with a black + . On the right panel the line profile corresponding to one of the marked pixels is shown (solid line) together with the average profile over the whole raster (dotted line). line profiles at the centre of which the largest radiance of the entire dataset was recorded (see Fig. 6, right panel).

\section{Summary and conclusion}

Our careful spectroscopic analysis of a large quiet area around Sun centre in the mid-transition region line O VI $\lambda 103.193 \mathrm{~nm}$ has revealed substantial mass flows (exceeding those associated with the average quiet Sun profiles) to be strongly correlated to the magnetic network. Line profiles from the regions of high magnetic flux concentration are Gaussian in shape and show bulk flows of the order of (10 to 30$) \mathrm{km} \mathrm{s}^{-1}$, either upwards or downwards directed. This seems to indicate mass flows on scales larger than the instrumental spatial resolution $\left(\approx 1.5^{\prime \prime}\right)$.

The non-Gaussian line profiles typical of the explosive events (EEs) testify, on the other hand, the presence of flows on scales smaller than the spatial resolution. Several explosive events were, hence, found in the observed area through a search for the non-Gaussian line profiles. We have estimated an event rate of $\approx 2500 \mathrm{~s}^{-1}$ over the whole Sun. We find that the kinetic energy and enthalpy fluxes associated with EEs do not play a significant role in the energy budget of the outer solar atmosphere. EEs are associated with mass flow patterns often larger than the area characterised by the non-Gaussian line profiles. However, although they are located along the network, EEs seem to avoid regions with strong magnetic flux concentrations. Regions that, although often characterised by relevant mass flows, show nearly Gaussian line profiles. These results could support the idea of a multi-component transition region characterised by large coronal funnel rooted at the strong flux concentrations and by a series of small cold loop that outline the magnetic network (as depicted by Dowdy et al. 1986). The majority of the coronal funnels would be the bases of large coronal loops, as also sketched by Peter (2001).

Mass flows seem to be present in all these structures, with those in the larger loops being characterised by subsonic speeds (at least close to the footpoints) and scales larger than the spatial resolution. In the smaller loops, small-scale supersonic flows (witnessed by the EE-like line profiles) seem instead a frequent feature. In, at least, one case (see Fig. 4) we find evidence that EE-like line profiles are clearly associated to a supersonic siphon-like flow in a loop structure. Another two possible cases have also been found. These flows seem to be triggered by pressure imbalances at the footpoints of small $\left(\approx 10^{\prime \prime}-15^{\prime \prime}\right)$ TR loops. However, only perhaps three such cases ( 3 couples of EEs locations) could be found out of $\approx 50$, thus siphon-like flows are likely to explain only a minority of the observed EEs.

As discussed in Sect. 1, EE-like profiles are believed to be the spectral signature of bi-directional jets generated by magnetic reconnection between oppositely directed magnetic field lines (Innes et al. 1997). Chae et al. (2000) considered bidirectional jets to occur as a result of a collision of a network and an internetwork flux thread. At the intersection point, the two flux threads are almost anti-parallel, forming an angle that is greater than $90^{\circ}$. An alternative model (Chae 1999) involves a two-step reconnection process. According to this model, the initial reconnection occurs low in the atmosphere with the formation of magnetic islands. These islands are observed as $\mathrm{H} \alpha$ up-flow events. The magnetic islands are annihilated by overlying magnetic field lines through a second reconnection that, being a fast reconnection process, produces the bi-directional jets. Such a model could explain the observation (Madjarska \& Doyle 2002) that EEs are first observed in chromospheric lines then in TR lines. It may also be consistent with the observed three to five minute recurrence rate of explosive events (Chae et al. 1998; Ning et al. 2004). The latter authors noted that this period is very close to the period of chromospheric and TR oscillations and suggested that initial reconnection low in the atmosphere could be triggered at a particular phase of a wave or oscillation.

EEs have some common aspects with spicules. In fact, spicules, like EEs, are related to the chromospheric network (Beckers 1968, 1972; Athay 1976; Suematsu 1998). The number of observed $\mathrm{H} \alpha$ spicules present on the Sun at any time is around $6 \times 10^{4}$, if only spicules higher than $5 \mathrm{Mm}$ are considered (observed heights are between 6.5 and 9.5 Mm). The number increases by one order of magnitude if smaller spicules are also considered (Beckers 1972). An average lifetime of $\approx 600 \mathrm{~s}$ (Beckers 1972) yields a birthrate between $2 \times 10^{-17}$ and $2 \times 10^{-16} \mathrm{~m}^{-2} \mathrm{~s}^{-1}$, indicating that between 2 and $20(0.7$ and $6.7 \mathrm{~s}^{-1}$ ) spicules should be present in our observed FOV (compared to around 50 EEs). Finally, we note that the upward directed mass fluxes in Fig. 3 cover about the $1.6 \%$ of the observed area. This value is very close to the $\approx 1 \%$ of the solar surface covered by spicules (Beckers 1968, 1972; Athay 1976). 


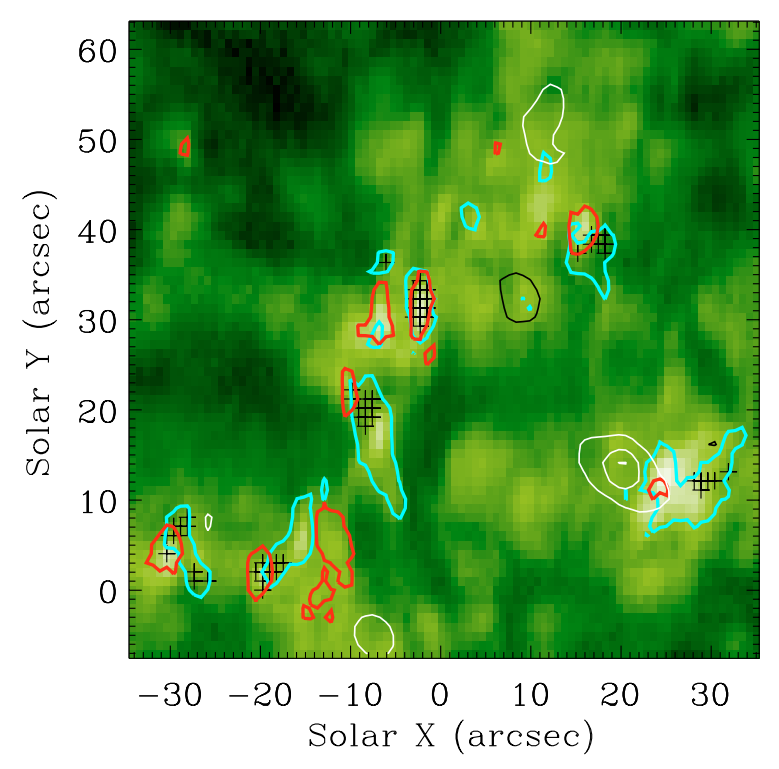

Fig. 7. Close-up of the area within box $\mathrm{C}$ in Fig. 3. Isocontours are defined as in Fig. 3. The locations where non-Gaussian line profiles were found are marked with a black + .

Wilhelm (2000) hypothesised that the rearrangement of the magnetic field lines following a reconnection process (shown by the EE-like profiles) could lead to the lifting of plasma, thus generating a spicule. In this case, an EE could be the first stage in the formation of a spicule. In most of our cases, blue and red patches seem to originate from a given EE location but with the blue pattern generally more extended. This means that the reconnection process affects an area much larger than that characterised by non-Gaussian line profiles. Figure 7 is a close-up of the area within box $\mathrm{C}$ in Fig. 3 and shows several examples of flow patterns associated to EEs. Particularly interesting is the velocity structure around $(X=-8, Y=20)$. In this case the explosive event is located at the base of $a \approx 15^{\prime \prime}$ long blueshifted structure that could be related to the upward apparent motions observed in spicules. The red contour could be directly related to the plasma accelerated downward from the EE location while the more extended blue pattern could be due to the upward accelerated plasma only at the base of the pattern (where the EE-like profiles are observed), while at larger distances it could be due to plasma lifted by the reconnecting field lines as suggested by the spicule model outlined by Wilhelm (2000) in his Fig. 8a-d. Another example of a flow pattern associated to a spicule could be that shown on the right panel of Fig. 6.

From a general comparison of the properties of these two types of events, we feel that our results indicate a possible link between the occurrence of the EEs and the formation of spicules. However, further high spectral and spatial resolution UV space observations in TR and, possibly, chromospheric lines (of a quality comparable to the data presented here), combined with simultaneous high spatial resolution images at several wavelengths across the $\mathrm{H} \alpha$ line are necessary to give a definitive answer on whether or not EEs and spicules are effectively related phenomena.
Acknowledgements. The authors thank D. E. Innes for fruitful discussion and K. Wilhelm for careful reading of the manuscript and for useful comments and suggestions. Many thanks also to the referee, V. Hansteen, for his comments that improved the manuscript. D.B. is grateful to the CPA, K. U. Leuven University for providing facilities during his stay at Leuven. D.B.'s work is partially supported by the ESA PRODEX project (ESA/Contract No. 14815/00/NL/SFe(IC)). Research at Armagh Observatory is grant-aided by the N. Ireland Dept. of Culture, Arts and Leisure. The SUMER project is financially supported by DLR, CNES, NASA, and PRODEX. SOHO is a mission of international cooperation between ESA and NASA.

\section{References}

Athay, R. G. 1976, The Solar Chromosphere and Corona: Quiet Sun (Dordrecht: Reidel)

Beckers, J. M. 1968, Sol. Phys., 3, 367

Beckers, J. M. 1972, ARA\&A, 10, 73

Bewsher, D., Parnell, C. E., \& Harrison, R. A. 2002, Sol. Phys., 206, 21

Boris, J. P., \& Mariska, J. T. 1982, ApJ, 258, L49

Bray, R. J., \& Loughhead, R. E. 1974, The Solar Chromosphere (London: Chapman and Hall)

Brekke, P., Kjeldseth-Moe, O., \& Harrison, R. A. 1997, Sol. Phys. 175,511

Brković, A., Solanki, S. K., \& Rüedi, I. 2001, A\&A, 373, 1056

Brueckner, G. E., \& Bartoe, J.-D. F. 1983, ApJ, 272, 329

Chae, J. 1999, in High Resolution Solar Physics: Theory, Observations and Techniques, ed. T. R. Rimmele, K. S. Balasubramaniam, \& R. R. Radick, Provo, ASP Conf. Ser., ASP, 183, 375

Chae, J., Wang, H., Lee, C.-Y., Goode, P. R., \& Schühle, U. 1998, ApJ, 497, L109

Chae, J., Wang, H., Goode, P. R., Fludra, A., \& Schühle, U. 2000, ApJ, 528, L119

Cook, J. W., Brueckner, G. E., Bartoe, J.-D. F., \& Socker, D. G. 1984, Adv. Space Res., 4, 59

Dere, K. P. 1992, in Solar Wind Seven: Proc. of the 3rd COSPAR Coll., ed. E. Marsch, \& R. Schwenn, 11

Dere, K. P., \& Mason, H. E. 1993, Sol. Phys., 144, 217

Dere, K. P., Bartoe, J.-D. F., \& Brueckner, G. E. 1989, Sol. Phys., 123, 41

Dere, K. P., Bartoe, J.-D. F., Brueckner, G. E., et al. 1991, J. Geophys. Res., 96, 9399

Dowdy, J. F. Jr., Rabin, D., \& Moore, R. L. 1986, Sol. Phys., 105, 35

Doyle, J. G., Madjarska, M. S., Dzifćáková, E., \& Dammasch, I. E. 2004, Sol. Phys., 221, 51

Feldman, U., Doschek, G. A., \& Mariska, J. T. 1979, ApJ, 229, 369

Harrison, R. A. 1997, Sol. Phys., 175, 467

Harrison, R. A., Sawyer, E. C., Carter, M. K., et al. 1995, Sol. Phys., 162,233

Harrison, R. A., Lang, J., Brooks, D. H., \& Innes, D. E. 1999, A\&A, 351,1115

Innes, D. E., Inhester, B., Axford, W. I., \& Wilhelm, K. 1997, Nature, 386,811

Kjeldseth-Moe, O., \& Brekke, P. 1998, Sol. Phys., 182, 73

Lemaire, P., Wilhelm, K., Curdt, W., et al. 1997, Sol. Phys., 170, 105

Madjarska, M. S., \& Doyle, J. G. 2002, A\&A, 382, 319

Madjarska, M. S., \& Doyle, J. G. 2003, A\&A, 403, 731

Mariska, J. T. 1988, ApJ, 334, 489

Mariska, J. T., \& Boris, J. P. 1983, ApJ, 267, 409

McClymont, A. N. 1989, ApJ, 347, L47

McClymont, A. N., \& Craig, I. J. D. 1987, ApJ, 312, 402

Müller, D. A. N., Hansteen, V. H., \& Peter, H. 2003, A\&A, 411, 605 
Ning, Z., Innes, D. E., \& Solanki, S. K. 2004, A\&A, 419, 1141

Orlando, S., Peres, G., \& Serio, S. 1995a, A\&A, 294, 861

Orlando, S., Peres, G., \& Serio, S. 1995b, A\&A, 300, 549

Peter, H. 2001, A\&A, 374, 1108

Peter, H., \& Brković, A. 2003, A\&A, 403, 287

Porter, J. G., \& Dere, K. P. 1991, ApJ, 370, 775

Reale, F., Peres, G., \& Serio, S. 1996, A\&A, 316, 215

Reale, F., Peres, G., \& Serio, S. 1997, A\&A, 318, 506

Robb, T. D., \& Cally, P. S. 1992, ApJ, 397, 329

Rüedi, I., Solanki, S. K., \& Rabin, D. 1992, A\&A, 261, L21

Ryutova, M. P., \& Tarbell, T. D. 2000, ApJ, 541, L29

Scherrer, P. H., Bogart, R. S., Bush, R. I., et al. 1995, Sol. Phys., 162, 129

Spadaro, D., Antiochos, S. K., \& Mariska, J. T. 1991, ApJ, 382, 338

Suematsu, Y. 1998, in Solar Jets and Coronal Plumes, ed. T.-D. Guyenne (Noordwijk: ESA Publication Division), ESA SP-421, 19
Teriaca, L., Madjarska, M. S., \& Doyle, J. G. 2001, Sol. Phys., 200, 91

Teriaca, L., Madjarska, M. S., \& Doyle, J. G. 2002, A\&A, 392, 309

Thomas, J. H., \& Montesinos, B. 1991, ApJ, 375, 404

Vernazza, J. E., Avrett, E. H., \& Loeser, R. 1981, ApJS, 45, 635

Warren, H. P., \& Winebarger, A. R. 2000, ApJ, 535, L63

Winebarger, A. R., Emslie, A. G., Mariska, J. T., \& Warren, H. P. 2002, ApJ 565, 1298

Wilhelm, K. 1997, in Fifth SOHO workshop: the Corona and Solar Wind Near Minimum Activity, ed. A. Wilson (Noordwijk: ESA Publication Division), ESA SP-404, 17

Wilhelm, K. 2000, A\&A, 360, 351

Wilhelm, K., Curdt, W., Marsch, E., et al. 1995, Sol. Phys., 162, 189 Wilhelm, K., Lemaire, P., Curdt, W., et al. 1997, Sol. Phys., 170, 75

Withbroe, G. L. 1983, ApJ, 267, 825 